\title{
Use of domperidone in canine visceral leishmaniasis: gaps in veterinary knowledge and epidemiological implications
}

\author{
Bruno L Travi ${ }^{1 /+}$, Guadalupe Miró ${ }^{2}$ \\ ${ }^{1}$ University of Texas Medical Branch, Department of Internal Medicine - Division of Infectious Diseases, Galveston, Texas, USA \\ ${ }^{2}$ Universidad Complutense de Madrid, Veterinary Faculty, Animal Health Department, Madrid, Spain
}

A pivotal strategy to decrease the risk of visceral leishmaniasis in humans is to control the infection and disease progression in dogs, the domestic reservoir of Leishmania infantum (L. chagasi). Immunotherapy is a viable approach to treat sick dogs because cell-mediated immunity is the principal defense mechanism against $L$. infantum. Domperidone is an immune-stimulatory drug increasingly used in veterinary medicine as a prophylactic or immunotherapeutic agent. Domperidone treatment has shown to prevent overt disease or improve the clinical condition of infected dogs. However, veterinarians should be aware of the potential cardiotoxicity of domperidone when given together with drugs that inhibit CYP450s liver enzymes or those that prolong the QT interval. On the other hand, learning whether domperidone treatment significantly decreases dog infectivity to sand fly vectors is of capital importance since this result should have a palpable impact on the infection risk of humans living in regions endemic for visceral leishmaniasis.

Key words: domperidone - dogs - visceral leishmaniasis

A pivotal strategy to decrease the risk of visceral leishmaniasis in humans is to control the infection and disease progression in dogs, which are the principal reservoirs of Leishmania infantum (L. chagasi). Current efforts to control the dispersion of this important zoonosis focusing on dogs as complement of insecticide spraying have been unsuccessful leading, principally, to the expansion of canine visceral leishmaniasis (CVL) in South American countries. Instead of placing emphasis on the controversial strategy of culling infected dogs, new approaches to control transmission that are more humane need to be selected. Deltamethrin-impregnated collars and different spot-on insecticides have shown high repellent efficacy against sand fly vectors. ${ }^{(1)}$ In Europe, both preventive approaches are feasible while in Latin America they face logistical and economical hurdles for its implementation. $(2,3)$ Vaccines are showing promising results but additional field trials are required to understand better the epidemiological implications of its utilization, mostly in areas of high $L$. infantum transmission. . $^{(1,4)}$

Therefore, antileishmanial treatment of dogs is still a valid option since it delays disease progression that is associated with increased transmission to sand flies. ${ }^{(5,6,7)}$ The treatment of CVL is complex, frequently resulting in recurrent disease related with high parasite burdens. (8) Combination of antimonials with allopurinol is currently the first line therapy used in most countries. ${ }^{(9,10)}$ In Brazil, miltefosine was approved for treatment of dogs infected with $L$. infantum in spite published information does not strongly support its utilization. ${ }^{(11,12)}$

doi: 10.1590/0074-02760180301

BLT and GM contributed equally to this work.

+Corresponding author: brltravi@utmb.edu

Received 25 June 2018

Accepted 2 October 2018
Cell-mediated immunity is the principal defense mechanism to prevent or maintain L. infantum infection under control. ${ }^{(13,14)}$ For this reason, immunotherapy is being considered as a viable approach to treat sick dogs. ${ }^{(15)}$ Domperidone (Motilium $\AA$, Leishguard $\AA$ or the generic drug) is an immune-stimulatory agent recommended in veterinary medicine as a prophylactic or immunotherapeutic agent, either as monotherapy or in combination with antileishmanial drugs. ${ }^{(16)}$ Although, few published studies are available detailing domperidone beneficial or untoward effects, its utilization is becoming increasingly popular in veterinary practice. A better understanding of its mode of action and drug interactions will maximize its efficacy and decrease the potential health risks to dogs at risk of $L$. infantum infection.

Domperidone is a benzimidazole derivative with selective dopamine D2 receptor antagonist activity. ${ }^{(17)}$ In humans, it is used as an anti-emetic, a gastro-kinetic drug or to increase milk production. ${ }^{(18)}$ In Veterinary medicine, domperidone could be used as a gastric pro-kinetic drug. (19) The drug induces an increase of prolactin serum level as a secondary effect. This hormone, which is excreted from the pituitary gland and generated by lymphocytes, is considered to be a pro-inflammatory cytokine. It stimulates the cellular immunity (Th1) by increasing the production of INF- $\gamma$, IL2, IL12, and TNF- $\alpha .^{(20)}$

A clinical trial in 70 naturally infected $\operatorname{dog} s$ indicated that domperidone significantly improved the clinical condition of animals. Clinical improvement was observed in 86\% (24/28) sick dogs, which was accompanied by stable antibody levels in $57.1 \%$ of animals. (21) Furthermore, at 90 days post-treatment, all infected dogs regardless of their initial clinical condition showed no clinical signs of disease and had negative or significantly lower antileishmanial antibody titers. The cellmediated immunity improved in dogs that were at different disease stages as determined by cell proliferation assays and Leishmania skin testing.(21) Unfortunately, no control group of untreated dogs was included in the 
study. This weakened the accuracy of domperidone results since it is known that a variable proportion of untreated dogs could remain asymptomatic or revert from sick to subclinical infection. ${ }^{(22)}$

Another clinical trial in an area highly endemic for CVL evaluated the capacity of domperidone to prevent development of disease. ${ }^{(23)}$ In this study, one group of healthy, seronegative dogs $(n=44)$ was treated with domperidone $(0.5 \mathrm{mg} / \mathrm{kg} /$ day for 30 days, every 4 months) while a second similar group $(n=46)$ was left untreated. The clinical-serological evaluation done after exposure to two sand fly seasons showed that a significantly ( $\mathrm{p}<$ $0.001)$ larger proportion of dogs treated with domperidone remained clinically healthy (89\%) compared with untreated controls (52\%). Authors defined this status as "protection" but the parasitological status of "protected dogs" was not assessed either by microscopy or molecular methods. Therefore, the impact of domperidone treatment on parasite burden in target organs (spleen, bone marrow, lymph nodes and/or skin) was not determined.

The label of veterinary domperidone (Leisguard ${ }^{\mathrm{TM}}$ ) does not recommend its use with cabergoline (dopamine agonist), dopamine and cimetidine, omeprazol or similar drugs. However, no trials to evaluate its interactions with other drugs have been performed. Consequently, we should not consider this drug as a supplement and have to be cautious with its administration. Personal experience (GM) have found some side effects associated with domperidone administration such as polyuria, dysorexia, vomiting and diarrhea. There are lingering questions regarding other aspects of domperidone therapy that require an in-depth discussion within the scientific and veterinary community. The treatment schedule for dogs recommended by the manufacturer of the veterinary product is $0.5 \mathrm{mg} / \mathrm{kg} /$ day for 30 days every three months, which is a low-dose regimen with few side effects. Since the recommendation of the manufacturer for dogs living in endemic areas is the administration of domperidone for three months each year, it would be important to gather more data on the potential short- and long-term clinical impact of this treatment in dogs of different age, breed and clinical condition.

The literature in humans indicated that some precautions need to be taken when using domperidone. A human population-based case-control study confirmed the higher risk of sudden cardiac death [OR (adj)] 11.4 (95\% CI 1.99, 65.2) when taking high doses of domperidone, e.g. $>30 \mathrm{mg} /$ day $(>0.4-0.5 \mathrm{mg} / \mathrm{kg} /$ day $) .{ }^{(24)}$ While the recommended dose of domperidone in dogs falls within the safe range, it is important to consider two different situations that may lead to life-threatening arrhythmias. Domperidone is known to prolong the QT interval and this untoward effect could be synergized if other drugs with a similar side effect are co-administered to the animal. ${ }^{(25)}$ Table includes a partial list of drugs of veterinary use with known or suspected QT prolongation activity.

Another circumstance in which domperidone concentration could inadvertently increase is when dogs concomitantly receive drugs that have strong cytochrome P450 inhibitory activity. Domperidone has low bioavailability when given through the oral route but its absorption increases in the presence of CYP3A1 and P-gp inhibitors. ${ }^{(26)}$ Domperidone is principally metabolized by CYP3A4 (CYP3A12 or CYP3A26 are the canine orthologs) and the interaction with drugs that inhibit this detoxification enzyme may increase domperidone concentration exposing the animal to cardiotoxic levels (QT prolongation). ${ }^{(27)}$ Ketoconazole, itraconazole and erythromycin are examples of veterinary drugs that inhibit CYP450's, and therefore should be avoided when dogs are under domperidone treatment. ${ }^{(28,29)}$ Other drugs with similar CYP450 inhibitory capacity are cimetidine, fluoroquinolones, chloramphenicol and phenobarbital. ${ }^{28}$ Before domperidone implementation, additional attention should be paid to conditions such as the age, breed and hormonal status of the animal. These variables have been associated with distinct detoxifying capacity of liver enzymes. ${ }^{(30)}$ Furthermore, the cardiological status, principally in older dogs or breeds prone to suffer heart conditions (i.e. boxer) must be considered. ${ }^{(31)}$

From the epidemiological standpoint, the impact of domperidone treatment on dog infectivity to sand fly vectors is still an open question. It is presumed that infected but clinically healthy dogs are at lower risk of transmitting $L$. infantum to sand flies compared with sick dogs. This has been demonstrated in studies carried out in Latin America upon feeding colonized Lutzomyia longipalpis sand flies on infected dogs (xenodiagnosis). . $^{(5,6,7)}$ However, earlier studies in Spain suggested that asymptomatic dogs have the capacity to transmit $L$. infantum to Phlebotomus perniciosus sand flies. ${ }^{(32)}$ Consequently, provided the latter results are the norm, xenodiagnosis studies like those previously used to evaluate the efficacy of antileishmanial drugs still need to be performed after domperidone treatment. ${ }^{(33,34)}$ This will determine whether treated dogs that are subclinically infected could act as reservoir hosts of visceral leishmaniasis.

TABLE

Generic names of veterinary drugs with QT interval prolongation

\begin{tabular}{lccc}
\hline Albuterol & Dopamine & Isoproterenol & Pseudoephedrine \\
Amantadine & Ephedrine & Methadone & Quinidine \\
Amiodarone & Epinephrine & Methylphenidate & Sotalol \\
Chlorpromazine & Erythromycin & Norepinephrine & Terbutaline \\
Disopyramide & Felbamate & Ondansetron & \\
Dobutamine & Granisetron & Procainamide &
\end{tabular}

Modified from Yap and Camm, 2003. ${ }^{(35)}$ 
General conclusions - Domperidone usage suggests that dogs benefit from its immunomodulatory effects, leading to protection against $L$. infantum infection or improvement of the clinical condition of infected individuals. Evaluation of the risks of side effects related to age, breed, drug interactions, concomitant endocrinopathies and cardiac fitness of dogs should precede domperidone administration. Veterinarians could make an essential contribution to pharmacovigilance, provided they consistently report to local drug agencies all the side effects observed during their clinical practice. The availability of these reports allows regulators to monitor the safety and efficacy of veterinary products once they reach the market. Importantly, domperidone assessment falls within the realm of "one health" since its utilization has impact on the health of both animal and human populations. Future efforts should be placed in defining the capacity of domperidone-treated dogs to participate as reservoir hosts of visceral leishmaniasis.

Provided domperidone demonstrates to reduce significantly the risk of treated dogs to transmit $L$. infantum to vectors, its large-scale implementation could be considered. Since domperidone acts as an immunostimulant with not known direct activity against the parasite, it sub-utilization would not carry the risk of developing drug resistance of L. infantum. In Europe, dog-owners are recommended to use the veterinary product, which is conveniently formulated for animal administration $(\approx 15$ $€ /$ treatment). In Latin America, the principal advantages are the low cost of the generic drug and its oral administration. The generic drug in Brazil costs approximately 0.08 USD per $10 \mathrm{mg}$ tablet; a $10 \mathrm{~kg}$-dog would require 15 tablets per treatment (1.20 USD) with a total cost of 3.60 USD for the three recommended treatments/year. An aspect to consider is the need for additional wellpowered trials in dog populations that could confirm the published results and meet the approval requirements of health authorities in each endemic country. While domperidone preliminary data look encouraging, its positive impact depends heavily on the government's commitment and resources to distribute the drug as well as achieving compliance of dog owners of endemic areas. Nevertheless, removal of free-roaming dogs will keep playing a critical role of control campaigns.

\section{REFERENCES}

1. Miro G, Petersen C, Cardoso L, Bourdeau P, Baneth G, SolanoGallego L, et al. Novel areas for prevention and control of canine leishmaniosis. Trends Parasitol. 2017; 33(9): 718-30.

2. Reithinger R, Coleman PG, Alexander B, Vieira EP, Assis G, Davies $C R$. Are insecticide-impregnated dog collars a feasible alternative to dog culling as a strategy for controlling canine visceral leishmaniasis in Brazil? Int J Parasitol. 2004; 34(1): 55-62.

3. Leite BMM, Solcà MS, Santos LCS, Coelho LB, Amorim LDAF, Donato LE, et al. The mass use of deltamethrin collars to control and prevent canine visceral leishmaniasis: a field effectiveness study in a highly endemic area. PLoS Negl Trop Dis. 2018; 12(5): e0006496.

4. Grimaldi Jr G, Teva A, dos-Santos CB, Santos FN, Pinto ID, Fux $B$, et al. Field trial of efficacy of the Leish-tec(R) vaccine against canine leishmaniasis caused by Leishmania infantum in an endemic area with high transmission rates. PLoS One. 2017; 12(9): e0185438.
5. Travi BL, Tabares CJ, Cadena H, Ferro C, Osorio Y. Canine visceral leishmaniasis in Colombia: relationship between clinical and parasitologic status and infectivity for sand flies. Am J Trop Med Hyg. 2001; 64(3-4): 119-24.

6. da Costa-Val AP, Cavalcanti RR, Gontijo NF, Michalick MS, Alexander B, Williams P, et al. Canine visceral leishmaniasis: relationships between clinical status, humoral immune response, haematology and Lutzomyia (Lutzomyia) longipalpis infectivity. Vet J. 2007; 174(3): 636-43.

7. Michalsky EM, Rocha MF, Lima ACR, Franca-Silva JC, Pires MQ, Oliveira FS, et al. Infectivity of seropositive dogs, showing different clinical forms of leishmaniasis, to Lutzomyia longipalpis phlebotomine sand flies. Vet Parasitol. 2007; 147(1-2): 67-76.

8. Baneth G, Koutinas AF, Solano-Gallego L, Bourdeau P, Ferrer L. Canine leishmaniosis-new concepts and insights on an expanding zoonosis: part one. Trends Parasitol. 2008; 24(7): 324-30.

9. Miro G, Cardoso L, Pennisi MG, Oliva G, Baneth G. Canine leishmaniosis - new concepts and insights on an expanding zoonosis: part two. Trends Parasitol. 2008; 24(8): 371-7.

10. Solano-Gallego L, Miró G, Koutinas A, Cardoso L, Pennisi MG, Ferrer L, et al. LeishVet guidelines for the practical management of canine leishmaniosis. Parasite Vectors. 2011; 4(1): 86.

11. Andrade HM, Toledo VP, Pinheiro MB, Guimaraes TM, Oliveira $\mathrm{NC}$, Castro JA, et al. Evaluation of miltefosine for the treatment of dogs naturally infected with $L$. infantum ( $=$ L. chagasi) in Brazil. Vet Parasitol. 2011; 181(2-4): 83-90.

12. Manna L, Corso R, Galiero G, Cerrone A, Muzj P, Gravino AE. Long-term follow-up of dogs with leishmaniosis treated with meglumine antimoniate plus allopurinol versus miltefosine plus allopurinol. Parasite Vectors. 2015; 8: 289.

13. Solano-Gallego L, Morell P, Arboix M, Alberola J, Ferrer L. Prevalence of Leishmania infantum infection in dogs living in an area of canine leishmaniasis endemicity using PCR on several tissues and serology. J Clin Microbiol. 2001; 39(2): 560-3.

14. Maia C, Campino L. Cytokine and phenotypic cell profiles of Leishmania infantum infection in the dog. J Trop Med. 2012; 2012.

15. Masihi K. Immunomodulatory agents for prophylaxis and therapy of infections. Int J Antimicrob Agents. 2000; 14(3): 181-91.

16. Lanaro E. Efficacy of a combined therapy whith meglumine antimoniate and domperidone for treatment of canine leishmaniosis. In: 73o Congresso Internazionale Multisala SCIVAC; 2011.

17. Barone JA. Domperidone: a peripherally acting dopamine2-receptor antagonist. Ann Pharmacother. 1999; 33(4): 429-40.

18. Reddymasu SC, Soykan I, McCallum RW. Domperidone: review of pharmacology and clinical applications in gastroenterology. Am J Gastroenterol. 2007; 102(9): 2036.

19. Woosley KP. The problem of gastric atony. Clin Tech Small Anim Pract. 2004; 19(1): 43-8.

20. Hinterberger-Fischer M. Prolactin as pro-inflammatory cytokine - considerations on consolidated immunotherapy after high dosage therapy. Acta Med Austriaca Suppl. 2000; 52: 16-20.

21. Gómez-Ochoa P, Castillo J, Gascón M, Zarate J, Alvarez F, Couto C. Use of domperidone in the treatment of canine visceral leishmaniasis: a clinical trial. The Vet J. 2009; 179(2): 259-63.

22. Falqueto A, Ferreira AL, dos Santos CB, Porrozzi R, da Costa MVS, Teva A, et al. Cross-sectional and longitudinal epidemiologic surveys of human and canine Leishmania infantum visceral infections in an endemic rural area of southeast Brazil (Pancas, Espirito Santo). Am J Trop Med Hyg. 2009; 80(4): 559-65. 
23. Sabaté D, Llinás J, Homedes J, Sust M, Ferrer L. A single-centre, open-label, controlled, randomized clinical trial to assess the preventive efficacy of a domperidone-based treatment programme against clinical canine leishmaniasis in a high prevalence area. Prev Vet Med. 2014; 115(1): 56-63.

24. van Noord C, Dieleman JP, van Herpen G, Verhamme K, Sturkenboom MC. Domperidone and ventricular arrhythmia or sudden cardiac death: a population-based case-control study in the Netherlands. Drug safety. 2010; 33(11): 1003-14.

25. Ehrenpreis ED, Roginsky G, Alexoff A, Smith DG. Domperidone is commonly prescribed with QT-interacting drugs: review of a community-based practice and a Postmarketing adverse drug event reporting database. J Clin Gastroenterol. 2017; 51(1): 56-62.

26. Athukuri BL, Neerati P. Enhanced oral bioavailability of domperidone with piperine in male wistar rats: involvement of CYP3A1 and P-gp inhibition. J Pharm Pharm Sci. 2017; 20: 28-37.

27. Boyce MJ, Baisley KJ, Warrington SJ. Pharmacokinetic interaction between domperidone and ketoconazole leads to QT prolongation in healthy volunteers: a randomized, placebo-controlled, doubleblind, crossover study. Br J Clin Pharmacol. 2012; 73(3): 411-21.

28. Trepanier LA. Cytochrome P450 and its role in veterinary drug interactions. Vet Clin Small Anim Pract. 2006; 36(5): 975-85.
29. Isoherranen N, Kunze KL, Allen KE, Nelson WL, Thummel KE. Role of itraconazole metabolites in CYP3A4 inhibition. Drug Metab Dispos. 2004; 32(10): 1121-31.

30. Martínez-Jiménez CP, Jover R, Donato MT, Castell JV, GomezLechon MJ. Transcriptional regulation and expression of CYP3A4 in hepatocytes. Curr Drug Metab. 2007; 8(2): 185-94.

31. Meurs KM, Spier AW, Miller MW, Lehmkuhl L, Towbin JA. Familial ventricular arrhythmias in boxers. J Vet Int Med. 1999; 13(5): 437-9.

32. Guarga JL, Lucientes J, Peribáñez MA, Molina R, Gracia MaJ, Castillo JA. Experimental infection of Phlebotomus perniciosus and determination of the natural infection rates of Leishmania infantum in dogs. Acta Trop. 2000; 77(2): 203-7.

33. Ribeiro RR, Moura EP, Pimentel VM, Sampaio WM, Silva SM, Schettini DA, et al. Reduced tissue parasitic load and infectivity to sand flies in dogs naturally infected by Leishmania (Leishmania) chagasi following treatment with a liposome formulation of meglumine antimoniate. Antimicrob Agents Chemother. 2008; 52(7): 2564-72.

34. Miro G, Galvez R, Fraile C, Descalzo MA, Molina R. Infectivity to Phlebotomus perniciosus of dogs naturally parasitized with Leishmania infantum after different treatments. Parasite Vectors. 2011; 4: 52.

35. Yap YG, Camm AJ. Drug induced QT prolongation and torsades de pointes. Heart. 2003; 89(11): 1363-72. 